\title{
Use of bacteriophages in the treatment of extensively drug-resistant Pseudomonas aeruginosa septicemia in a patient with acute kidney injury - a case report
}

Serge Jennes, Maia Merabishvili, Patrick Soentjens, Kim Win Pang, Thomas Rose, Elkana Keersebilck, Olivier Soete, Pierre-Michel François, Simona Teodorescu, Gunther Verween, Gilbert Verbeken, Daniel De Vos, Jean-Paul Pirnay

Burn Wound Center, Queen Astrid military hospital, Brussels, Belgium

A 62-year-old man was hospitalized for severe abdominal sepsis with disseminated intravascular coagulation, secondary to a diaphragmatic hernia with bowel strangulation. The patient had a prolonged hospital course complicated by gangrene, resulting in the amputation of the lower limbs and two fingers and the development of large necrotic pressure sores on the back. Three months later, the patient was transferred to the burn wound center of the Queen Astrid military hospital for surgical management of the pressure sores. Wound cultures on admission revealed MRSA, MDR P. aeruginosa, E. cloacae, Klebsiella pneumoniae and Candida albicans

colonisation. The patient developed septicemia with extensively drug-resistant (XDR), colistin-only-sensitive, $P$. aeruginosa. Intravenous (IV) colistin and sulfamethoxazole/trimethoprim combination therapy was started. Ten days later, the patient developed acute kidney injury, probably caused by drug-induced acute interstitial nephritis. The patient was in a coma and antibiotic therapy was stopped. Unfortunately, XDR $P$. aeruginosa septicemia re-emerged with positive hemocultures for three consecutive days. Upon expert advice and informed consent from the patient's family, IV and topical bacteriophage therapy were initiated under the umbrella of Art. 37

of the Declaration of Helsinki. Fifty $\mathrm{ml}$ of purified bacteriophage cocktail BFC1 (Merabishvili et al. 2009), containing two active $P$. aeruginosa bacteriophages in sodium chloride $0,9 \%$ at a concentration of $10^{7}$ plaque forming units (PFU) per ml were administered as

a 6 h IV infusion for 10 days. Wounds were washed with bicarbonate buffer and irrigated with $50 \mathrm{ml}$ BFC 1 every 8 h for 10 days.

Immediately, blood cultures turned negative, CRP levels dropped and the fever disappeared. Kidney function recovered after a few days. Hemodialysis was avoided and no clinical abnormalities related to the application of bacteriophages were observed. This is, as far as we know, the first documented report of intravenous bacteriophage monotherapy against $P$. aeruginosa septicemia in humans.

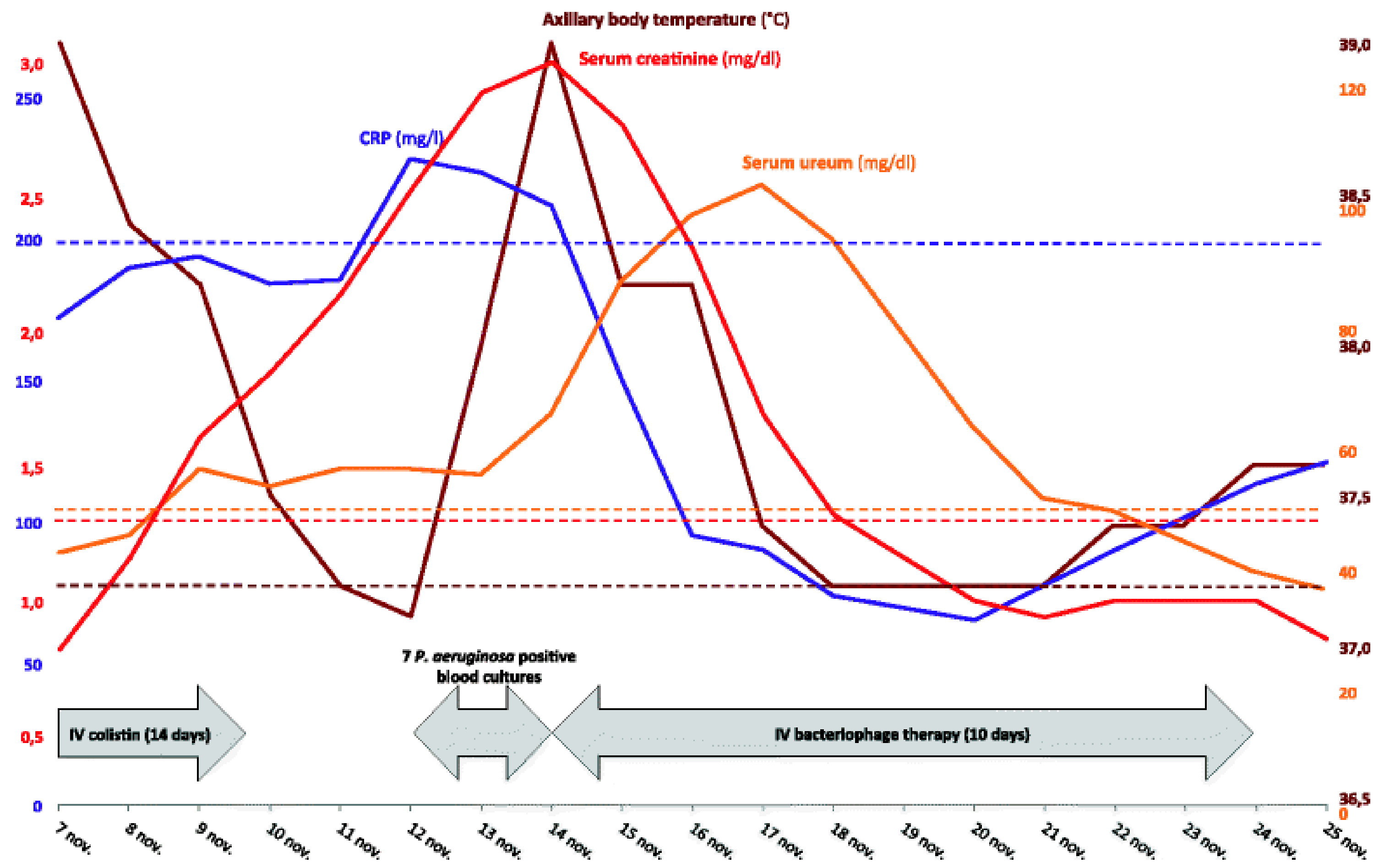

Figure 1. Timeline showing relevant clinical parameters and treatments. Dotted lines mark the upper threshold values. CRP C-reactive protein.

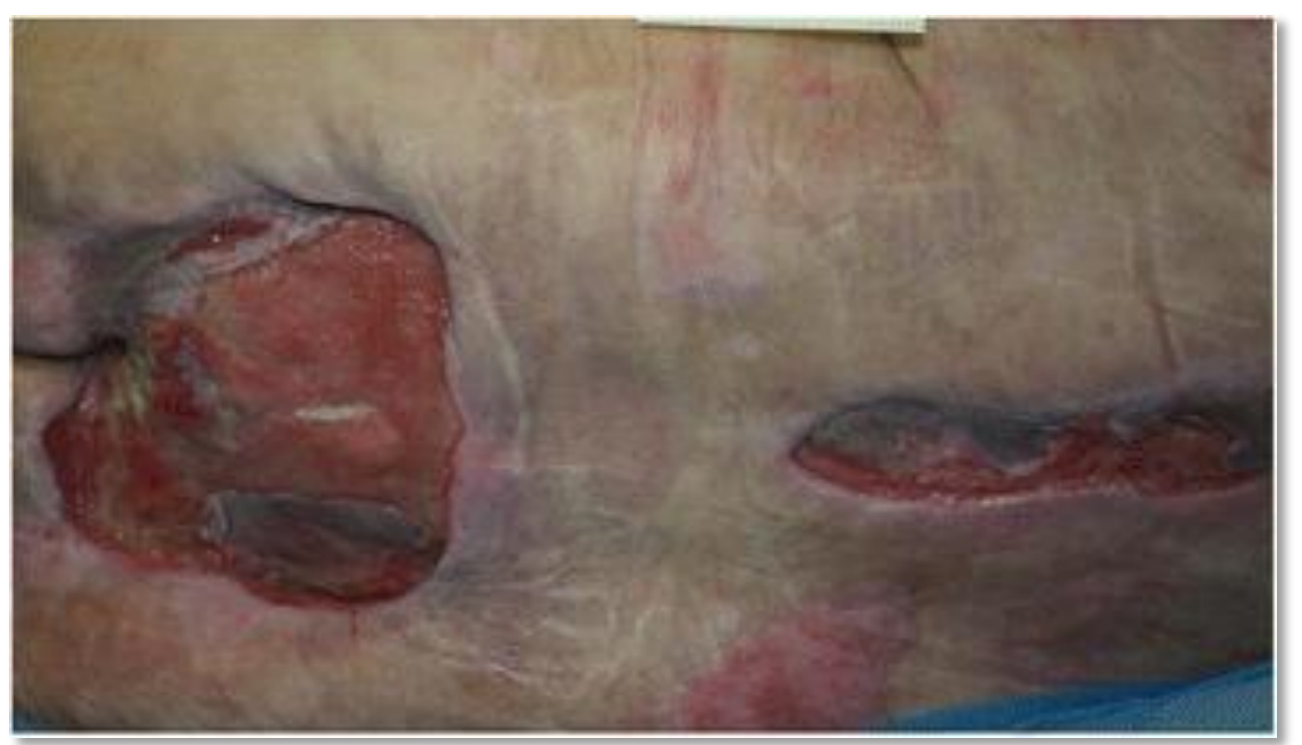

